\title{
Daily rhythm of salivary IL-1ß, cortisol and melatonin in day and night workers
}

\author{
Érica Lui Reinhardt ${ }^{\mathrm{a}, \mathrm{b}, *}$, Pedro Augusto Carlos Magno Fernandes ${ }^{\mathrm{c}}$, Regina Pekelmann Markus ${ }^{\mathrm{c}}$, and \\ Frida Marina Fischer ${ }^{\mathrm{a}}$ \\ ${ }^{a}$ Dept Environmental Health, School, School of Public Health, University of São Paulo, Av. Dr. Arnaldo, 715, \\ 01246-904, São Paulo, SP, Brazil \\ ${ }^{\mathrm{b}}$ Occupational Hygiene Division, Fundacentro, R. Capote Valente, 710, 05409-002, São Paulo, SP, Brazil \\ ${ }^{\mathrm{c} D e p a r t m e n t ~ o f ~ P h y s i o l o g y, ~ B i o s c i e n c e s ~ I n s t i t u t e, ~ U n i v e r s i t y ~ o f ~ S a ̃ o ~ P a u l o, ~ R . ~ d o ~ M a t a ̃ o, ~ T r a v e s s a ~ 14, ~ S a ̃ o ~ P a u l o, ~}$ \\ SP, Brazil
}

\begin{abstract}
Shiftwork-induced sleep deprivation and circadian disruption probably leads to an increase in the production of cytokines and dysregulation of innate immune system, respectively. This project aims evaluating changes in salivary IL- 1 beta, cortisol, and melatonin in night workers. Method. Two day and three night healthy workers participated in this study. Sleep was evaluated by actimetry and activity protocols. Saliva was collected at waking and bedtime the last workday and the following two days-off and was analyzed by ELISA. Results. Neither sleep duration nor efficiency showed any association with salivary IL-1beta. IL-1 beta levels were higher at waking than at bedtime during working days for all workers, but only one day and one night-worker maintained this pattern and hormone rhythms during days off. For this night worker, melatonin levels were shifted to daytime. A second one presented clear alterations in IL-1beta and hormone rhythms on days-off. Conclusions. Our preliminary results suggest that night work can disturb the variation pattern of salivary IL-1beta. No association of this variation with sleep was observed. It seems that disruption in hormone rhythms interfere with salivary IL-1beta production. ILlbeta production pattern seems to be maintained when rhythms are present, in spite of a shift in melatonin secretion.
\end{abstract}

Keywords: shiftwork; cytokines; biological rhythms

\section{Introduction}

Permanent night work can affect rhythmic secretion of melatonin and cortisol.

There is some evidence that shiftwork-induced sleep deprivation and circadian disruption can alter the production of inflammatory cytokines $[1,2]$ and disrupt the innate immune system [3], respectively.

This on going project aims evaluating changes in salivary level of IL-1ß, cortisol, and melatonin according to a work schedule of five fixed days or night shifts followed by two days off.

\section{Method}

Sleep parameters were estimated by actimetry and activity protocols for 10 consecutive days for five male healthy workers, three night (shift: 21:00-6:00) and two day workers (shift: 7:00-17:00) from a welding company located in São Paulo, SP, Brazil.

Saliva was collected at waking and bedtime the last workday (Friday) and in the following two daysoff (weekend). Analyses were performed by ELISA (eBioscience, San Diego, USA; IBL, Hamburg, Germany).

Data on sleep duration and sleep efficiency were submitted to $t$ tests. Associations between IL-1ß levels and sleep parameters were tested by Pearson's correlation tests.

\footnotetext{
* Corresponding author. E-mail: erica.reinhardt@fundacentro.gov.br
} 


\section{Results}

Table 1

Average results and standard deviations for IL-1ß, sleep duration and sleep efficiency for the last workday and the following two days-off.

\begin{tabular}{lllllll}
\hline & & \multicolumn{2}{l}{ IL-1ß levels $(\mathrm{pg} / \mathrm{mL})$} & & \\
& & Bedtime & Waking & Difference & Sleep Duration (min.) & Sleep Efficiency (\%) \\
\hline DW1 & Night sleep & $50.5 \pm 19.7$ & $298.8 \pm 39.5$ & 248.2 & $376.0 \pm 181.0$ & $81.8 \pm 11.0$ \\
DW2 & Night sleep & $169.4 \pm 103.9$ & $193.2 \pm 115.8$ & 23.8 & $310.0 \pm 30.4$ & $90.4 \pm 1.6$ \\
NW1 & Daytime sleep & $56.8 \pm 2.8$ & $244.2 \pm 3.7$ & 187.4 & $130.5 \pm 96.9$ & $44.0 \pm 20.3$ \\
& Night sleep & $71.1 \pm 47.9$ & $261.6 \pm 45.1$ & 190.6 & $208.0 \pm 203.6$ & $38.0 \pm 53.8$ \\
NW2 & Daytime sleep & $2.7 \pm 0.3$ & $163.2 \pm 147.0$ & 160.6 & $353.0 \pm 181.0$ & $77.1 \pm 15.1$ \\
& Night sleep & $39.6 \pm 9.9$ & $61.2 \pm 51.2$ & 43.2 & $422.5 \pm 13.4$ & $62.3 \pm 1.7$ \\
NW3 & Daytime sleep & 81.9 & 241.8 & 159.9 & 201.0 & 79.5 \\
& Night sleep & $118.0 \pm 138.0$ & $299.0 \pm 102.7$ & 181.0 & $487.0 \pm 1.41$ & $77.7 \pm 2.41$ \\
\hline
\end{tabular}

DW = day worker; NW = night worker; Pearson's correlation tests for IL-1ß levels, sleep duration, and sleep efficiency showed that these

variables are not associated.

Day workers are on this same day shift for 9.5 years on average, while night workers are in the same night shift for a shorter time, 3.5 years on average. All workers reported satisfaction with work shift, which they chose to work.

Regarding day workers, no significant differences were observed between total sleep duration on workdays and days off $(352.58 \pm 66.11 \mathrm{~min}$. and $360.88 \pm$ $33.41 \mathrm{~min}$, respectively) or between sleep efficiency for the same days $(88.29 \pm 2.82 \mathrm{~min}$. and $87.06 \pm$ 0.01 min., respectively). On the other hand, it seems that night workers tend to sleep more on weekends (398.08 $\pm 115.66 \mathrm{~min}$. for night sleep) than on working days $(283.56 \pm 28.97 \mathrm{~min}$.). Sleep efficiency of night workers shows virtually no difference when we compared workdays and days off $(75.39 \pm 4.90$ and

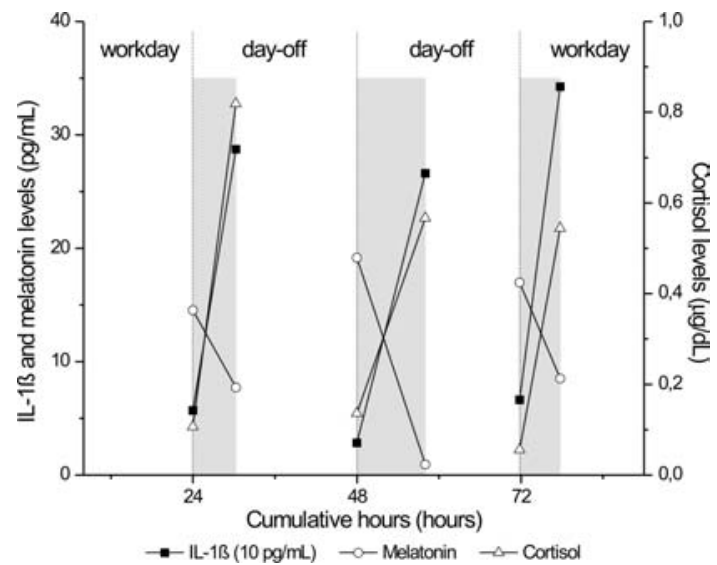

Fig. 1. Hormones and IL-1ß levels of Day Worker 1 . IL-1ß variation pattern, melatonin and cortisol rhythms on last workday were maintained on days-off. Shaded areas correspond to sleep time, and 24, 48 and 72 hours correspond to midnight.
$72.95 \pm 13.51$, respectively), although the sleep efficiency was worse than that of day workers.

As shown in Table 1, salivary IL-1ß levels were higher on awakening $(279.65 \pm 49.00 \mathrm{pg} / \mathrm{mL})$ than at bedtime $(52.83 \pm 29.92 \mathrm{pg} / \mathrm{mL})$ for all workers in the last day of work, regardless of work shift, time they went to bed, sleep efficiency or total sleep duration.

One day (Day Worker 1) and one night worker (Night Worker 1) maintained this variation pattern of higher levels on awakening and lower levels on bedtime for salivary IL-1 1 during days off (Figures 1 and 2 , respectively). For these two workers cortisol and melatonin rhythms observed on last workday were also maintained during days off.

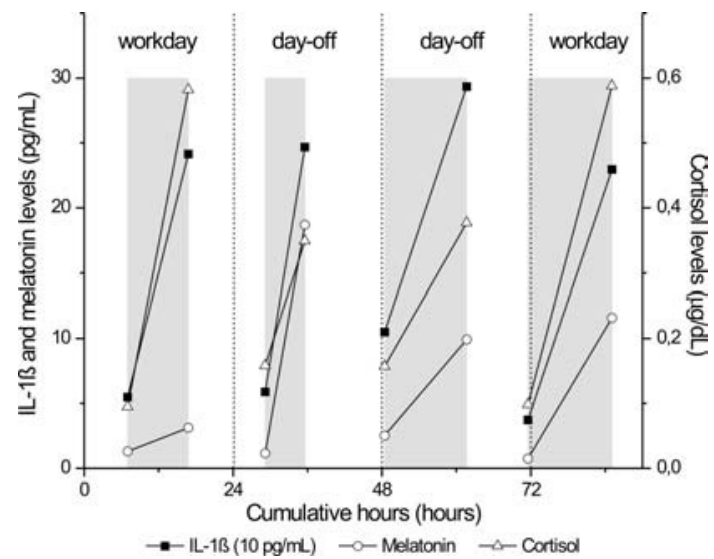

Fig. 2. Hormones and IL-1ß levels of Night Worker 1. IL-1ß variation pattern on last workday was maintained on days-off and melatonin secretion is shifted to daytime. Shaded areas correspond to sleep time, and 0, 24, 48 and 72 hours correspond to midnight. 


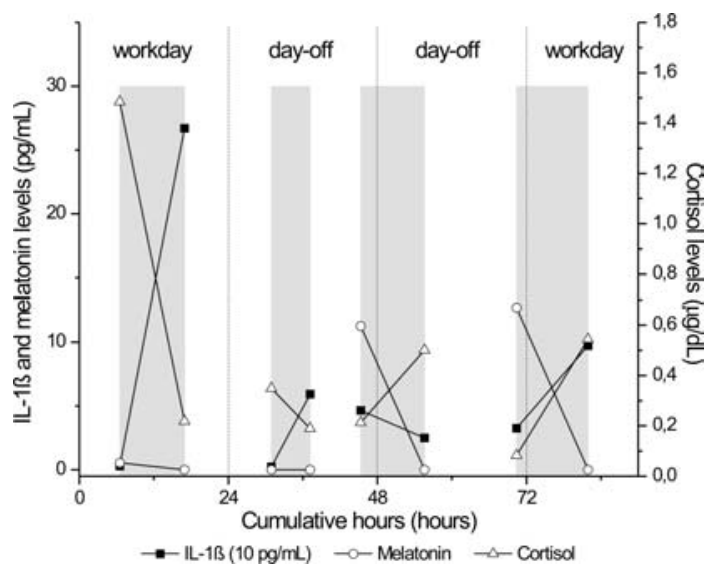

Fig. 3. Hormones and IL-1ß levels of Night Worker 2. This worker presented clear alterations in IL-1ß variation pattern, melatonin and cortisol rhythms. Shaded areas correspond to sleep time, and 0 , 24, 48 and 72 hours correspond to midnight.

Cortisol and melatonin rhythms of Day Worker 1 were the expected for individuals that work day shifts and sleep during nighttime. Night Worker 1 , however, shows a phase shift in both hormone rhythms, which causes melatonin secretion to coincide with daytime sleep and maintenance of cortisol secretion on awakening, which occurs later when compared to Day Worker 1.

A second night worker showed clear alterations in the variation pattern of salivary IL- $1 \beta$ and cortisol rhythm on days off (Figure 3). The secretion of melatonin in this worker apparently was not shifted to daytime sleep and maintained the expected rhythm for a person with a diurnal activity/rest cycle, in which melatonin secretion occurs during night sleep.

\section{Discussion}

Our preliminary results suggest that the variation pattern of salivary IL-1ß can be disturbed by night work. It also seems that disruption in melatonin and cortisol rhythms occur simultaneously with alterations in the salivary IL- $1 ß$ levels at waking and bedtime. Conversely, these levels seem to be maintained when the rhythms are present, in spite of the melatonin secretion shift to daytime. These results agree with findings of Castanon-Cervantes and colleagues [3], that disruption in circadian system affects directly the production of inflammatory cytokines. However, due to the small number of investigated subjects, it was not possible to establish a clear association between melatonin, cortisol and IL-1ß.
Apparently, production of IL-1ß is not associated with altered sleep, as reported in the literature [1,2]. It is possible that this association couldn't be observed due to the small size of the sample. Thus, further analyses are necessary to a better understanding of the effects of sleep on the production of salivary IL-1ß. Sleep data and saliva samples of additional participants are already being collected and analyzed.

\section{Acknowledgements}

We thank the research subject volunteers for their participation in this study and the owners and administrators of the welding company. This work was supported by Fundacentro and a grant from FAPESP (process number 2011/10503-4).

\section{References}

[1] A.A. Prather, A.L. Marsland, M. Hall, S.A. Neumann, M.F. Muldoon and S.B. Manuck, Normative variation in selfreported sleep quality and sleep debt is associated with stimulated pro-inflammatory cytokine production, Biol Psychol 82 (2009), 12-17.

[2] W.M.A. van Leeuwen, M. Lehto, P. Karisola, H. Lindholm, R. Luukkonen, M. Sallinen, M. Härmä, T. Porkka-Heiskanen and H. Alenius, Sleep restriction increases the risk of developing cardiovascular diseases by augmenting proinflammatory responses through IL-17 and CRP, PLoS ONE 4 (2009), e4589.

[3] O. Castanon-Cervantes, M. Wu, J.C. Ehlen, K. Paul, K.L. Gamble, R.L. Johnson, R.C. Besing, M. Menaker, A.T. Gewirtz and A.J. Davidson, Dysregulation of inflammatory responses by chronic circadian disruption, J Immunol 185 (2010), 579-805. 